\title{
A study of stress, displacements and strain in a stress concentrator using analytical calculus and Finite Element Method
}

Brizeida N. Ojeda G.

Mechanical Engineering Department.

EAFIT University

Medellín, Colombia

bojedag@eafit.edu.co

A study of stresses, displacements and strain, based on a classic solid mechanic's model, specifically a plate with hole, around which a stresses concentration, is presented. For this purpose, the stresses analysis was carried out in a hole concentrator subjected to tensile. The model's material was ASTM A36. The stresses were analytically calculated, through von Mises' theory. In addition, the analysis of the stresses using the finite element method (FEM) was carried out. Subsequently, displacements and unitary deformation were determined in the part. The results obtained report an error of $8.7 \%$ between the stresses of von Mises through analytical calculus and using the FEM.

Keywords: Stresses concentration, Displacements, Unit Strain, Finite Element Method. 


\section{Introduction}

Engineering designs are increasingly demanding, due to the challenges in which they are incurs in order to increase the efficiency of the results [1], for example the optimization of designs minimizing weight [2], and the use of new materials [3]. To do this, the engineer has seen the need to use computational tools that serve as support to predict the behavior of the designs posed [4]. The vast majority of the computational tools for engineering designs are based on the finite elements method [5]. The presence of holes, steps, slots for cotter pins, spaces for the placement of dowels, cause in the loaded part an increase in the stresses around the area of discontinuity, known as stress concentration [6]. These regions should have paid special attention when designing a mechanical element, which is why this phenomenon has been analyzed theoretically and experimentally; As a result, equations and diagrams have been obtained for different geometry and load types to determine the stresses concentration factors. Materials and methods

\section{Methodology}

\subsection{Initials considerations}

To develop the study, a rectangular cross-section plate is selected as a model of analysis, the geometric details of which are shown in Fig. 1., with hole in the center which causes that in the surrounding area is produced the phenomenon of stresses concentrations.

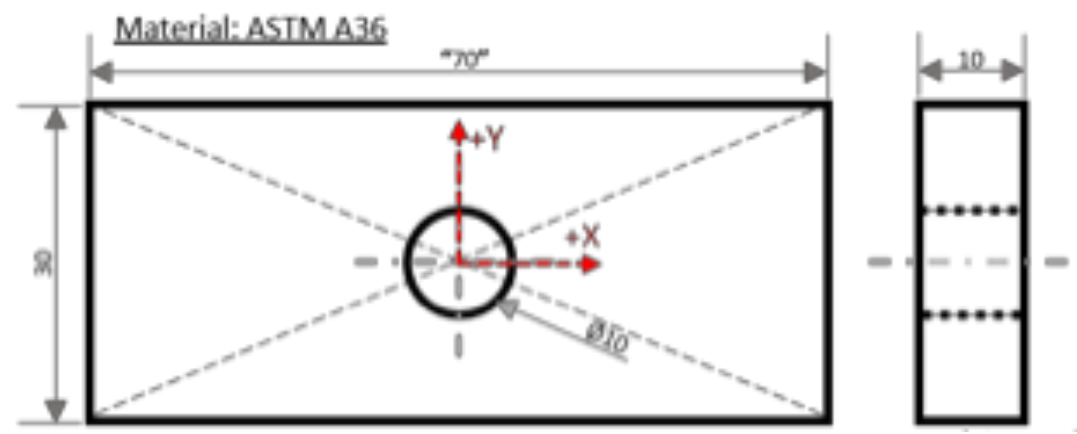

Fig. 1. Analysis Model: Rectangular cross-section plate geometry with stresses concentrations 
The plate is supposed to be steel ASTM A36, whose physical Young's modulus is es E $=2 \times 1011 \mathrm{~Pa}$. According to the configuration presented in the Fig. 1, It is intended to estimate the actual tensions distribution in a stress concentrator, along the $Y$ axis, carrying out the following procedure:

- $\quad$ For the analytical calculation the von Mises theory is chosen together with the Saint Venant principle.

- $\quad$ The piece modeling is done in the SolidWorks software for observation through FEM.

- After analyzing, by both methods, the margin of error is calculated between the results obtained.

- $\quad$ Finally, it is concluded on the results obtained from the analytical theory calculation and computational used, in order to determine the reliability of the results using the FEM in geometries with the presence of stress concentrators.

\subsection{Analytical calculus}

For the calculation of the stresses in points $C, D$ and $E$ (Fig. 2), three different stresses were taken into account. For the minimum stress, the $A-A$ area is taken, for the intermediate, the area B-B.

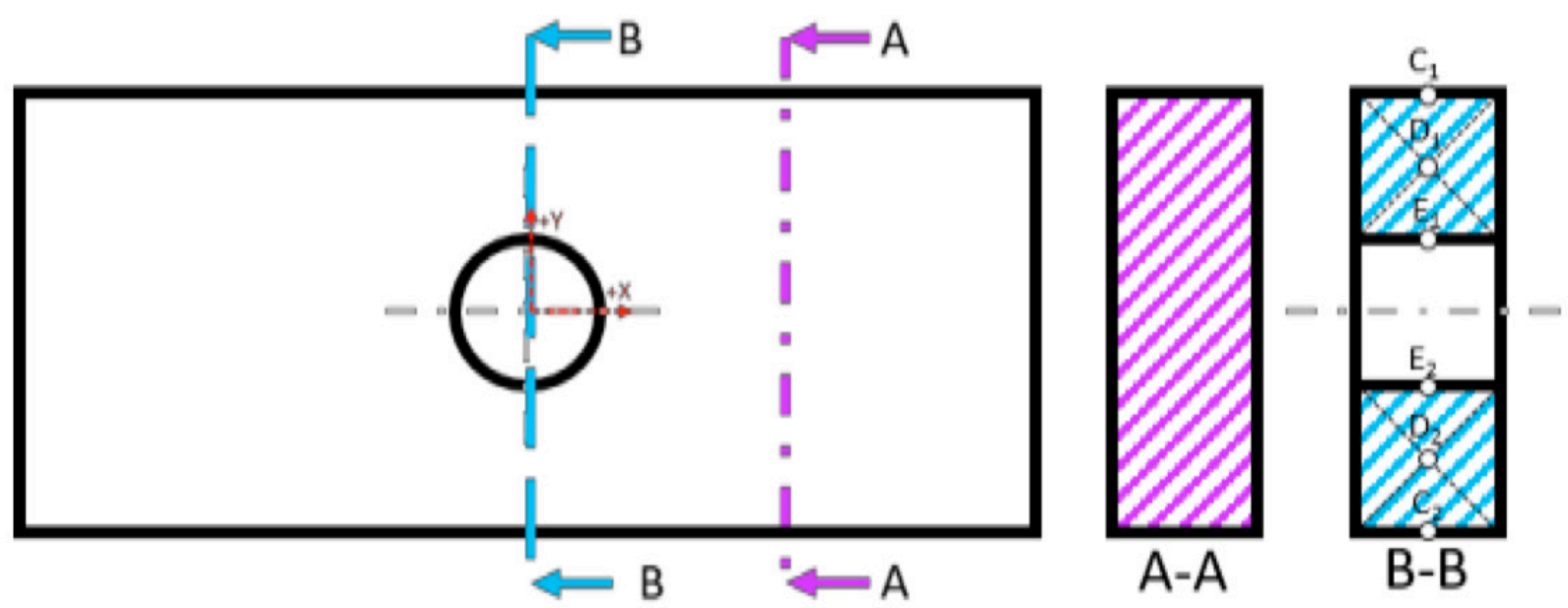

Fig. 2. Sections and points to evaluate on the plate 
For the calculation of the stress maximum, the equations 1, 2, 3 and 4 were used.

$$
\begin{aligned}
\sigma_{0} & =\frac{F}{A_{A-A}} \\
\sigma_{\text {Int. }} & =\frac{F}{A_{B-B}} \\
K_{t g} & =\frac{K_{t n}}{\left(1-\frac{\phi}{w}\right)} \\
\sigma_{\max } & =K_{t g} * \sigma_{0}
\end{aligned}
$$

The previously calculated stresses are used as described in Fig. 3.

The equations 5 and 6 are used for unit strain calculations.

$$
\begin{array}{cc}
\varepsilon_{\text {Max. }}=\frac{\sigma_{\text {Max. }}}{E} & \text { Eq. } 5 \\
\delta_{\text {Max. }}=L * \varepsilon_{\text {Max. }} & \text { Eq. } 6
\end{array}
$$

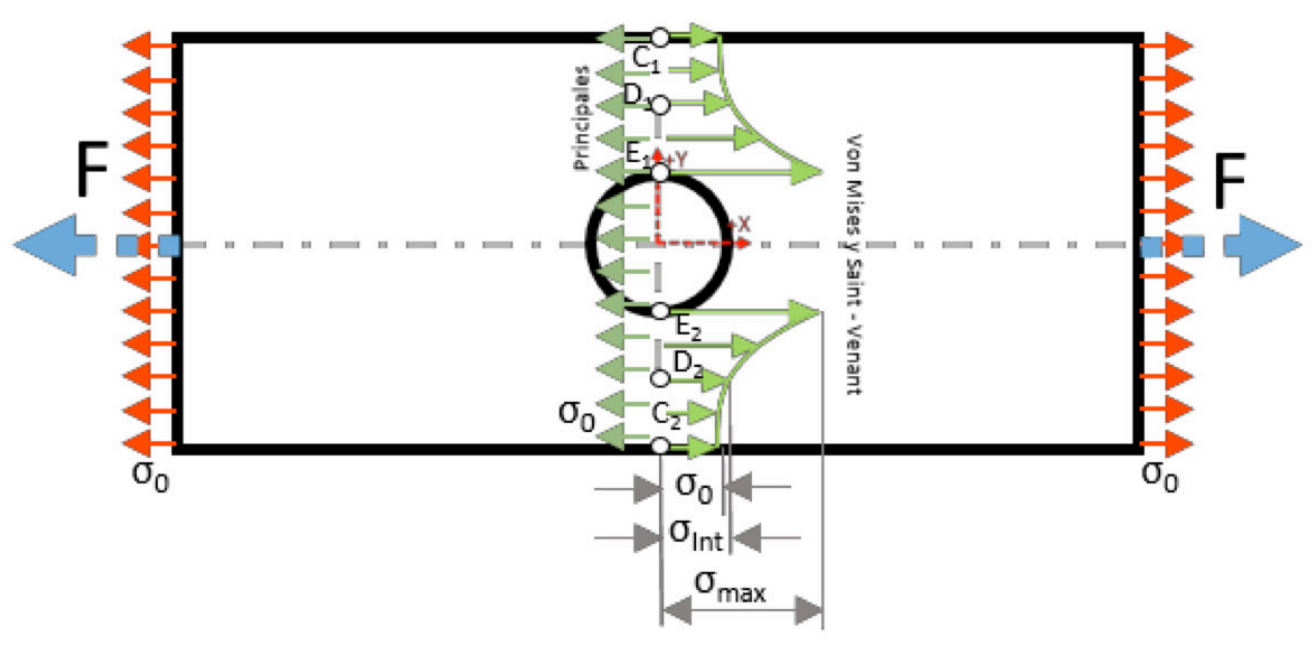

Fig. 3. Von Mises stresses on the plate 


\subsection{Calculations using FEM}

With the software used, the modelling is done with the dimensions of Fig. 1, the only assumed values were the length $70 \mathrm{~mm}$ and the position of the circle in the center of the rectangle; then extruded to the specified depth. As boundary conditions, a one side of the plate is fixed, and the other a force of $15 \mathrm{kN}$ in tension. In the results of the FEA, it is applied the given material, and later, a tracing of tensions to find the maximum value of exertions by von Mises, then a cut of section is made taking as reference the plane of the side view. In the process 15 nodes are selected in the vertical, in the zones of values of maximum stresses and the respective stress is found. For the cases of displacement and unit strain, the maximum values are found in the same way as the stresses were made. The Table 1 shows the comparison of the results obtained in the stresses with the two methods used, while Table 2, reports the unit strains and Table 3, the displacements, respectively.
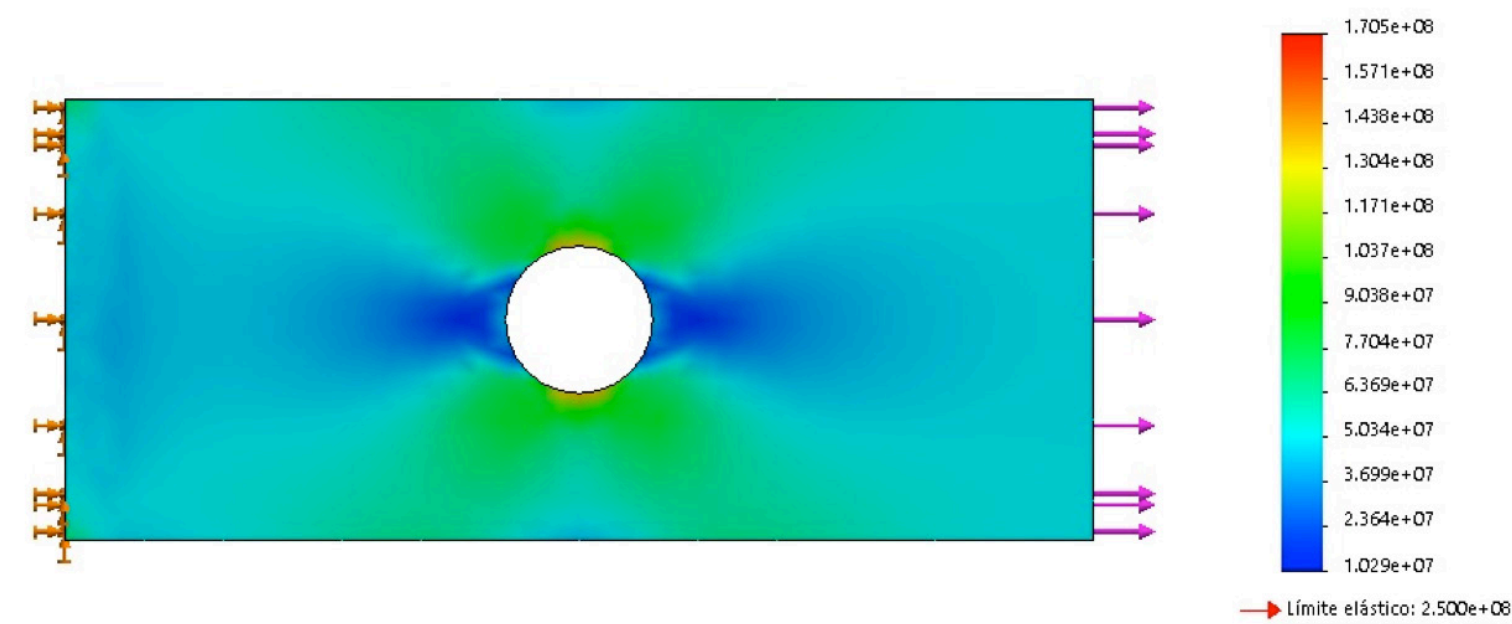

Fig. 4. Von Mises stress using FEM

Table 1. Results comparison and error percentage: Stress

\begin{tabular}{cccc}
\hline $\mathrm{y}(\mathrm{mm})$ & $\begin{array}{c}\text { von Mises (FEM) } \\
{[\mathrm{MPa}]}\end{array}$ & $\begin{array}{c}\text { von Mises (Analítico) } \\
{[\mathrm{MPa}]}\end{array}$ & \% Error \\
\hline 15 & 45,77 & 50,0 & 8,5 \\
10 & 72,42 & 75,0 & 3,4 \\
5 & 170,58 & 172,5 & 1,1 \\
-5 & 170,5 & 172,5 & 1,2 \\
-10 & 72,18 & 75,0 & 3,8 \\
-15 & 45,64 & 50,0 & 8,7 \\
\hline
\end{tabular}


Table 2. Results comparison and error percentage: Unit strain

\begin{tabular}{ccc}
\hline FEM & ANALYTICAL & \% Error \\
\hline & & \\
$5,974 \mathrm{E}-04$ & $8,625 \mathrm{E}-04$ & $30,74 \%$ \\
\hline
\end{tabular}

Table 3. Results comparison and error percentage: Displacement

\begin{tabular}{ccc}
\hline FEM & ANALYTICAL & \% Error \\
\hline 0,01967 & $6,038 \mathrm{E}-02$ & $67,42 \%$ \\
\hline
\end{tabular}

Diagram A in Fig. 5, the comparison between the two methods used, are showed.

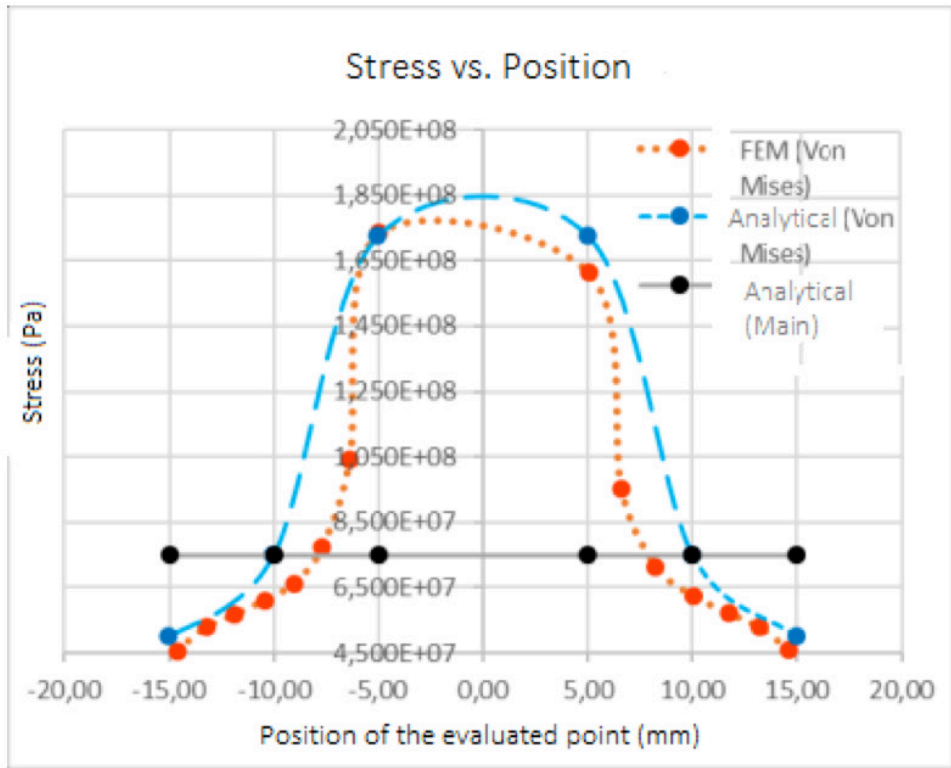

Fig. 5. FEM vs. Analytical

When observing Fig. 4, it can be verified that the stresses calculated by FEM, increase when approaching the center of the piece, this is due to the effects of the load in the central zone, which is the most prone to fail. That is why, as the analytical stress by von Mises took the Saint Venant principle as a reference, it is said that it was the closest to the real value.

With respect to Finite Element Analysis, it can be said that it is better to calculate by this method, because, when taking more nodes to evaluate, it makes a much more precise estimation of the effects of the load on the plate. From the results obtained, it can also be concluded that as the stresses are greater towards the center of the piece, these points should be examined at the time of analyzing it and if the given stress is greater than the stress of creep (to this case ? $Y=2,5 \times 108 \mathrm{~Pa}$ )ilt is said to fail. During this analysis, as the maximum stress is lower, it is said that it does not present any failure. 
With respect to the gross concentration factor, used to calculate the maximum stress starting from the minimum (Fig. 3), as its value is 3.45, it can be confirmed that the stresses at an end near the applied load are 3.45 times larger than the minimum value.

\section{Conclusions}

The use of a software based on the finite element method, is a good support for the realization of engineering designs that involve the determination of stress, displacement and strain in complex parts, especially with stress concentrators. Also, the software allows to determine the distribution of tensions in zones with concentrators of stresses with geometric form not defined analytically.

\section{References}

[1] Camacho, D., Hopper, R., Lin, G., \& Myers, B. (1997). An improved method for finite element mesh generation of geometrically complex structures with application to the skullbase. Journal of Biomechanics, 30(10), 1067-1070. Obtenido de https://doi. org/10.1016/S0021-9290(97)00073-0

[2] Asmussen, E., Peutzfeldt, A., \& Sahafi, A. (2005). Finite element analysis of stresses in endodontically treated, dowel-restored teeth. The Journal of Prosthetic Dentistry, 94(4), 321-329. Obtenido de https://doi.org/10.1016/j.prosdent.2005.07.003

[3] Leclerc, W., Haddad, H., \& Guessasma, M. (2019). DEM-FEM coupling method to simulate thermally induced stresses and local damage in composite materials. International Journal of Solids and Structures, 160(15), 276-292. Obtenido de https:// doi.org/10.1016/j.ijsolstr.2018.10.030

[4] Metan, S., Krishna, P., \& Mohankumar, G. (2014). FEM Model an Effective Tool to Evaluate Von Mises Stresses in Shoulder Joint and Muscles for Adduction and Abduction. Procedia Materials Science, 2090-2098.

[5] Preciado, A., \& Sperbeck, S. (2019). Failure analysis and performance of compact and slender carved stone walls under compression and seismic loading by the FEM approach. Engineering Failure Analysis, 96, 508-524. Obtenido de https://doi. org/10.1016/j.engfailanal.2018.11.009

[6] Budynas, R., \& Nisbett, K. (2015). Shigley's Mechanical Engineering Design. New York: Mc Graw Hil. 


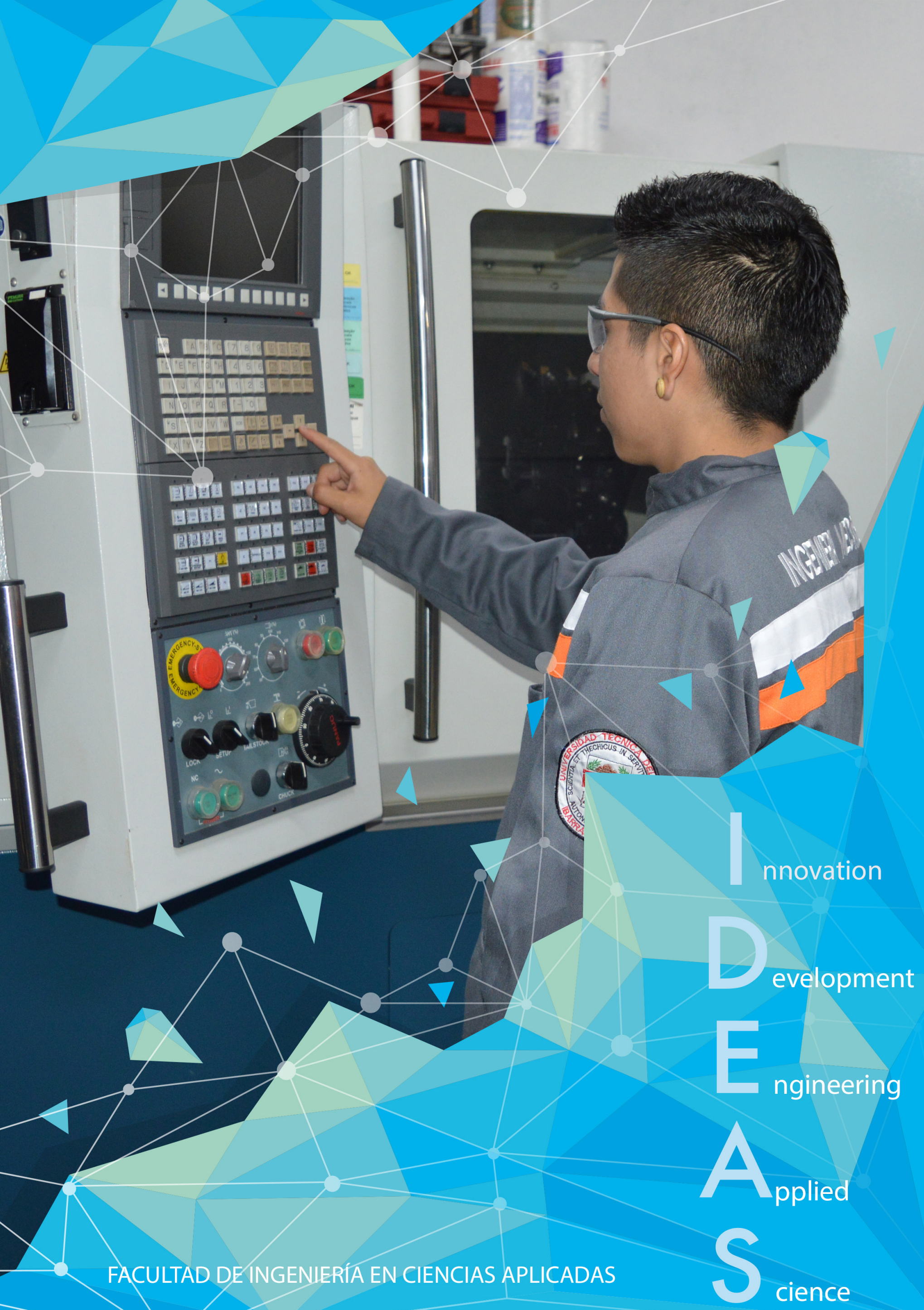

